\title{
Cleft lift technique with tension-free primary closure for repair of pilonidal sinuses: Three years experiences
}

\author{
Ayman Soliman, MD; Yasser Abdel Reheem, MD; \\ Ahmad Awad, MD; Osama El Shiekh, FRCS
}

Department of General Surgery, Ain Shams University, Cairo, Egypt.

\begin{abstract}
Objective: Cleft lift technique was performed in patients with chronic or recurrent pilonidal sinus (PS). It has been reported to provide better results than simple excision and closure in the midline. The aim of this retrospective study was to evaluate the results after introducing the tension-free flap as asymmetric cleft lift procedure in our hospital on a day care basis.

Patients and methods: Between June 2008 and July 2011, 236 patients (199 males, 37 females: median age 30 years; range, 18-46 years) underwent excision of the pilonidal sinus with tension-free primary closure in cleft lift technique. Patients were observed and followed up (126 cases (53.3\%) of these patients accepted a consultation in the outpatient clinic while 75 cases (31.7\%) were interviewed by phone) for the healing rate and reporting the postoperative criteria over 2 years.

Results: At follow up mean 24 (range 10-27) months after the operation, 169 (72.6\%) of the wounds were healed while recurrences were present in 32 (13.5\%) of the patients. Further results related to pre-, per- and postoperative conditions are discussed in this paper.

Conclusion: Cleft lift procedure results in a similar looking wound of the midline and a shallow cleft. It is very successful in the treatment of difficult recurrent sinuses, where the early results are promising.

Key words: Pilonidal sinuses, recurrences, asymmetric, flap, Bascom technique, cleft lift.
\end{abstract}

\section{Introduction:}

Pilonidal sinus disease (PSD) is commonly encountered in the surgical outpatient clinic. The incidence is 26/100.000 and the condition is most frequent among men in their third decade of life. A recent study indicates that traditional midline techniques for pilonidal sinus repair give a high wound infection rate, high recurrence rate, poor cosmetic results and a long time to healing. In a met- analysis comparing results from 74 publications on primary closure techniques in chronic pilonidal sinus repair the authors conclude that asymmetric or oblique closure techniques (Karydakis-flap, Bascoms procedure, oblique excision etc.) or fullthickness plasty techniques (rhomboid flaps, vy-plasty and z-plasty) provide better results than the simple closure technique in the natal midline.
In 1973, Dr. George Karydakis, in Greece, excised the sinus with a simple biconvex 'elliptical' excision just crossing the midline to excise the sinus. It was based $1-2 \mathrm{~cm}$ from the midline with excision down to the sacrum

The asymmetric or cleft lift technique with primary closure is recommended because they are considered easier to perform providing the same results as the full-thickness plasty techniques. Both Karydakis and Bascom have described asymmetric closure technique. Karydakis published his experience with a simple and successful operation to cure pilonidal sinus and presented the largest personal series in the world where he describes removal of all deep inflamed tissue and the fixation of the base of a mobilized, asymmetric thick flap to the sacrococcygeal fascia before skin closure. Bascom describes a thin skin flap mobilization leaving the deep 
inflamed tissue in place and skin closure only. Both methods share excellent results; Karydakis reports a less than $1 \%$ recurrence rate and Bascom reports a $100 \%$ healing rate after minor revisions or a second cleft lift in 9-10\% of his patients with refractory PSD.

Due to its simplicity we introduced the cleft lift technique with tension-free primary closure as our single procedure for all symptomatic, chronic pilonidal sinus disease. The aim of this retrospective study was to evaluate the results of our first series of these operations.

\section{Methods:}

All patients operated on with the cleft lift procedure from June 2008 to July 2011 were followed up in the outpatient clinic. If a patient declined physical examination, he or she was interviewed by phone if willing. We were able to contact 201 (85\%) of the 236 patients who were operated on during this time period. Patients were admitted to the study in Ain Shams University Hospital, Ain Shams Specialized Hospital (Egypt), Dr. Soliman Fakeeh Hospital and Armed Forces Hospital King Abdulaziz Naval Base Jubail (Saudi Arabia). All of these patients, 199 (84.3\%) men and 37 (15.6\%) women, accepted to take part in the study and were included. 126 cases (53.3\%) were seen in the outpatient clinic while 75 cases (31.7\%) declined physical examination and were interviewed by phone within the same timeframe, the rest 35 cases (14.8) did not show up after removal of stitches for follow up. The mean time period from operation to follow up was 24 (range 10-27) months Table(1).

The mean age at operation was 30 (range 18-46) years. 142 patients (60\%) were overweight $(\mathrm{BMI} \geq 25)$ and among these 36 $(25.3 \%)$ were obese $(\mathrm{BMI} \geq 30)$. The average BMI was 26.6 (range 18.3-34.0). Patients were questioned about pre- and postoperative conditions and physically examined for recurrent pilonidal sinus. The mean duration of the disease before the cleft lift technique operation was 10 months (range 6 - 16 months). 156 patients $(66 \%)$ of the included patients were primary disease cases whereas 80 (34\%) were recurrent cases after previous PSD operations Figure(1). Sixteen of the patients with recurrent disease had been operated on two or more times. These previous operations were drainage of abscesses (83.5\%) and wide excisions (16.5\%). All patients were operated on in a day care unit. Four of the 236 patients had to stay overnight because of nausea. Operations were performed under local anesthesia supplemented with intravenous analgetic medicaments in all but one patient who needed general anesthesia because of excessive pain.

The operation was performed with liberal usage of local anesthesia (and adrenaline), which was preferable to general anesthesia in the prone position. This was applicable in non- nervous patients if sinus was not too large. We gave plenty of time to insert LA slowly under IV sedation with fine needle. The flap was created first, usually $2 \mathrm{~cm}$ wide and $0.5 \mathrm{~cm}$ deep under the skin Figure(2). Then, buttock straps were removed from the edges of operating table and the skin flap gently pulled across the midline with skin hooks to see if the mark of the outer rim of the 'ellipse' has been made correctly on the skin, and to make adjustments to prevent tension on wound closure. The covering skin flap from the opposite side was undermined. Less may need to be excised from the top end where the cleft was shallower than lower down.

The outer limit of excision was then cut with the scalpel, but instead of going down to the sacrum, the fat was left and only skin and dermis were excised, until the sinus was reached. We then prefer to fully excise the sinus track rather than clean it. The flap was fit gently across the midline on the fat rolled in from the other side over the suction drain tube. Few fine PDS or vicryl sutures were used in the fat and did not need to be inserted down to the sacral fascia, unless it was a deep sinus and the fascia was exposed. The 'ellipse' should be marked out on the operating table by first marking two points on one side (the most diseased side), a distance of $1.5-2 \mathrm{~cm}$ from the midline and placed far enough apart to allow a gentle curve to be marked out to just 
cross the midline a few mms, to include the primary pit in the excision. For small sinuses, use $1.5 \mathrm{~cm}$ from the midline. The distance of maximum width of the 'ellipse' is twice the distance the upper and lower points are from the midline $(3-4 \mathrm{~cm})$. This is measured between the marked lines not between the midline and the outer edge. After probing the tracks to work out their extent, the use of methylene blue injection into the midline pit will enable all branches to be easily identified in case any are if severed or opened during the excision Figure(3).

To avoid a 'dog-ear' at the lower end or the wound tending to 'move' towards the midline, a $\mathrm{V}$ excision of skin can be taken off the lower end laterally. This will deviate the lower end a little further from the midline when skin is closed. A fine low-pressure suction drain tube is placed in the fat, brought out lateral to the wound. Allow no suture holes or drain hole to appear in the new midline. Use a subcuticular $3 / 0$ prolene suture loosely knotted as a loop (the wound lengthens when the patient sits, so loose suture needed to prevent a 'cheesecutter' effect). We support it with a few interrupted $3 / 0$ prolene sutures. IV antibiotic including metronidazole is given during the procedure. Drain was removed two or three days after the operation when patients came back for a post-operative visit and all sutures out on tenth day.

Patients are instructed for the postoperative care at home to keep the wound dry and to avoid prolonged pressure on the wound for about a week post-operatively. Those that went home with a drain are taught how to empty the drain every day until it is ready to be removed. Prophylactic antibiotics are administered intravenously (Metronidazole $1.5 \mathrm{~g}$ and Cefuroxime 1.5g) at the beginning of the operation and continued orally for the first five days after the operation (Metronidazole $0.5 \mathrm{~g} \times 3$ and Cephalexin 0.5g $\times 3$ ). No preoperative bowel preparation is performed. The patients were told to shower the wound after defecation if necessary. Paracetamol is recommended for postoperative pain treatment. All patients are enrolled in a study with telephone and/or office follow-up requested to monitor long-term outcomes.

\section{Results:}

At follow up 24 (10.1\%) patients were without symptoms or signs of the disease while recurrence was found in 9 (4\%) of the patients. The recurrences occurred on the average 4 months after the operation and in 2 of these patients relevant errors in the procedures were discovered: In one patient the nonabsorbable, intradermal suture was never removed. In another the suture was removed later than scheduled on day 21 instead of on day 10 as planned. For the other 7 recurrences no evident cause was revealed. Four (45\%) of the nine patients with recurrences after the cleft lift procedure had been previously operated for pilonidal abscesses with simple incisions and drainage whereas 7 of 169 (4.1\%) in the cured group were previously operated for this condition. None of the nine recurrences had been previously operated with more radical, potentially curative techniques as e.g. wide excision.

The complications after the cleft lift operation consisted of five patients (2.6\%) with hematoma requiring no further treatment, one patient $(1.3 \%)$ of infection where the wound healed 9 months after revision, and $24(10.7 \%)$ patients with residual open segments of their wounds after removal of the intradermal suture. Fourteen of these wounds healed spontaneously after a mean of 6.3 (range 3-12) months, while seven did not heal and were thus classified as recurrences at follow up. Three patients (33\%) of the recurrent pilonidal sinus openings were found caudally in the cicatrice while 2 (22\%) were located cranially Table(2).

The non-absorbable intradermal suture was removed after a mean of 12.2 days except from one patient where the suture was not removed. Patients were off work/school for 11.5 (range 3-60) days after the operation. At follow up on the average 12 months after the operation, 112 (47\%) patients could not recall to have suffered from any significant pain after the operation while 16 (6.7\%) felt pain for a median of 2.6 (range 1-14) days. 

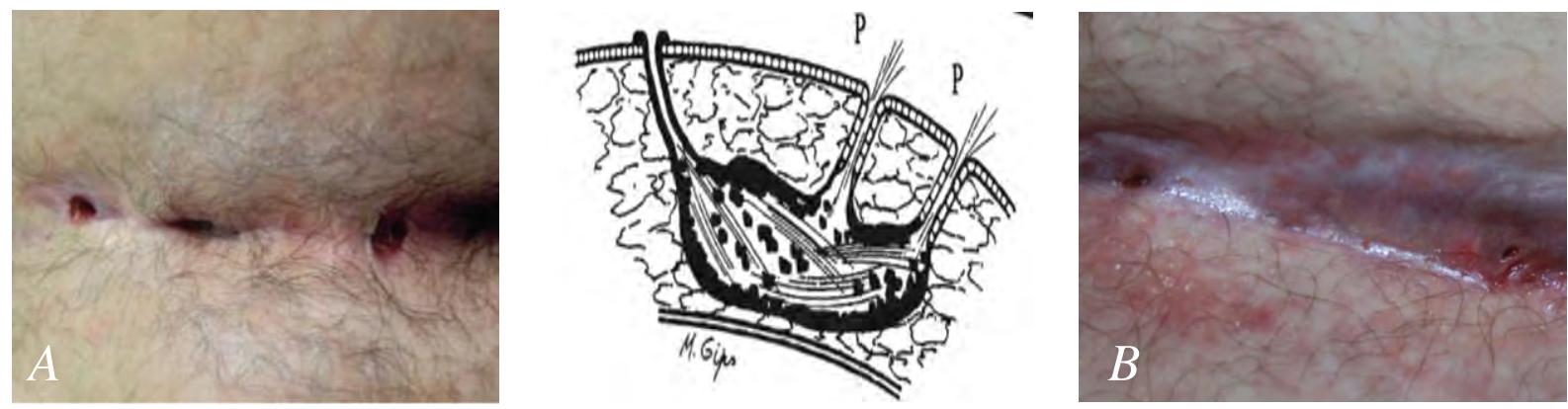

Figure (1): Pre-operative picture of patient with chronic, open sinuses (A) \& recurrent cases (B) of pilonidal disease.
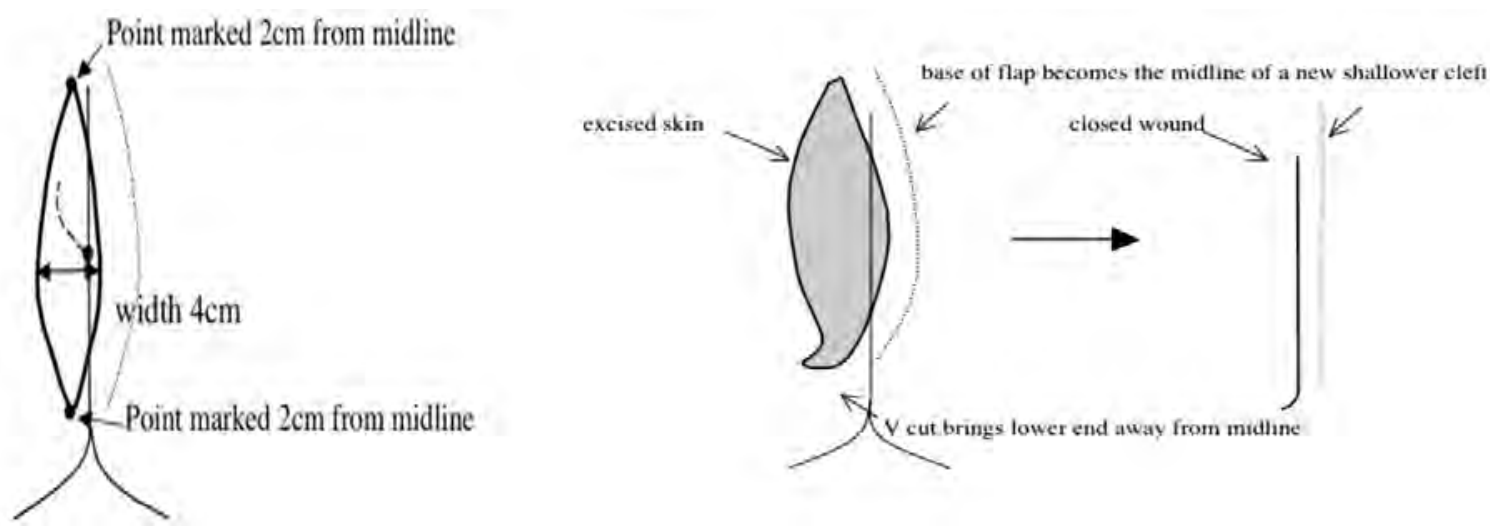

Figure (2): Cleft lift procedure where Point marked $2 \mathrm{~cm}$ from midline, width $4 \mathrm{~cm}$ and $0.5 \mathrm{~cm}$ deep under the skin.
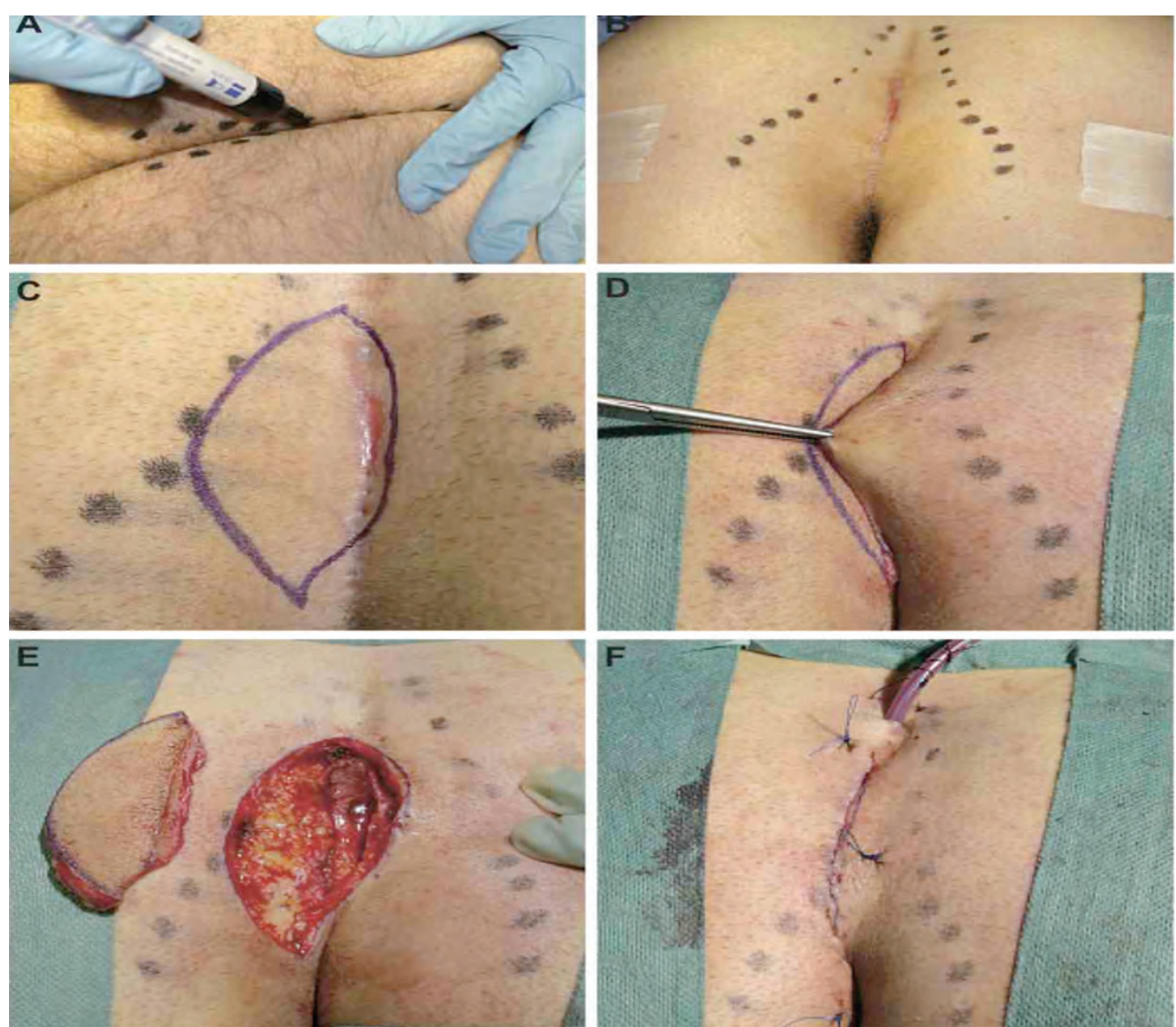

Figure (3): Cleft lift procedure; (A, B) Recurrent pilonidal sinus. (C, D) Incision mark around the sinus openings \& the incision line is a little off the midline. (E) Cut a flap; $2 \mathrm{~cm}$ wide, 1 $\mathrm{cm}$ deep \& full length of wound (F) Small drain is visible at the upper left of the photograph, Cut beyond and lateral to lower end (dealing with a dog ear). 
Table (1): Patients' characteristics and operative findings.

\begin{tabular}{|lc|}
\hline Characteristic & $\%$ \\
\hline Total number of patients: & 236 \\
- Male patients & $84.3 \%(199)$ \\
- Female patients & $15.7 \%(37)$ \\
\hline Age (years) & $20.93 .6(18-46)$ \\
\hline Duration of symptoms (months) & 10.016 .4 \\
\hline Presenting symptoms: & \\
- Pain & $89.7 \%$ \\
- Discharge & $61.9 \%$ \\
- Swelling & $57.3 \%$ \\
- Acute Suppuration & $12.7 \%$ \\
\hline Primary pilonidal sinus & $156(66) \%$ \\
Recurrence (Previous operative management): & $80(34) \%$ \\
- Drainage procedure & $83.5 \%$ \\
- Excision & $1.3 \%$ \\
\hline Operative findings: & $16.5 \%$ \\
\hline - Chronic pilonidal disease & $32.6 \%$ \\
- Pilonidal abscess & $2.3(0-16)$ \\
\hline Mean number of pits and openings: & \\
- Patient with midline pits only. & \\
- Patient with pits and 1 fistula. & \\
\hline
\end{tabular}


Table (2): Clinical outcomes of cleft lift operation for pilonidal disease.

\begin{tabular}{|lc|}
\hline Outcome & No. Of patients (\%) \\
\hline Total number of patients & 236 \\
\hline Patients with postoperative attendance & $226(95.7)$ \\
\hline Postoperative analgesics use & $143(60.9)$ \\
\hline Reported fever & $16(7.0)$ \\
\hline Clinical verified infection & $3(1.3)$ \\
\hline Postoperative bleeding & $5(2.6)$ \\
\hline Complete healing & $169(72.6)$ \\
- Healing within 2 weeks. & $28(17.3)$ \\
- $\quad$ Healing within 3 weeks. & $42(24.0)$ \\
- Healing within 4 weeks. & $99(58.7)$ \\
\hline Failed healing (rejected complementary treatment) & $7(2.9)$ \\
\hline
\end{tabular}

\section{Discussion:}

In spite of high incidence of pilonidal disease affecting young population and the prolonged disabling period caused by it, surgeons have not reached to the best treatment for this condition. No matter what the cause, patients with pilonidal disease can have multiple, recurring bouts of infection and discomfort. Some undergo repeat drainages of abscesses. Some undergo many operations in an attempt to remove the affected area. Too often, these operations fail to fully eradicate the disease.

In 1973, Dr. George Karydakis, in Greece, published his experience with a simple and successful operation to cure pilonidal sinus and later on presented the largest personal series in the world. He excised the sinus with a simple biconvex 'elliptical' excision only just crossing the midline to excise the sinus. It was based $1-2 \mathrm{~cm}$ from the midline with excision down to the sacrum. A thick flap was then created by undercutting the midline side of the wound. This flap was advanced across the midline to meet the other side of the wound with two layers of catgut sutures to the fat around a drain tube. The wound was then closed with skin sutures. Karydakis believed and taught that hair insertion is the cause of pilonidal sinus and attributed his extremely low recurrence rate of $(1 \%)$ to two facts. These are: (a) the whole wound is placed away from the midline (recurrences always occur in the midline) and (b) the resulting new natal cleft is shallower (so hairs do not collect so readily).

Despite these good results, the Karydakis operation has been criticised for taking too much fat, and for placing sutures into the midline sacral fascia (often causing pain). Most of his patients required general anesthesia and stayed in hospital a day or more. Dr Karydakis was trying to develop ways to simplify the operation for day surgery. Excision of the pilonidal sinus and laying the tract open to allow healing by secondary intention has been described as an option to ensure that the cavity has adequate drainage. This avoids a wound infection after primary closure. Consider laying the tract open when the primary closure is not free of tension. Even after excision of the pilonidal 
sinus down to healthy presacral fascia, the wound is still considered contaminated. Both aerobic and anaerobic organisms are found in $50-70 \%$ of wounds. The disadvantages of laying the tract open are the inconvenience to the patient, with frequent dressing changes, and close observation of the wound to ensure proper wound healing.

Skin flaps have also been described to cover a sacral defect after wide excision. Similarly, this keeps the scar off the midline and flattens the natal cleft. The potential complications include loss of skin sensation in the flap, which is observed in more than $50 \%$ of patients, and necrosis of the flap edges. Again, primary healing is achieved in $90 \%$ of cases. 'Cleft lift procedure' was first introduced by Dr. John Bascom in Oregon.

The cleft lift technique emphasized the circulation of hairs from a midline primary pit and through secondary openings. So secondary openings a distance from the main track and weren't included in the excision (e.g., by $\mathrm{V}$ cuts on one side or both sides of the wound to close and result in a T-shaped wound) Figure(2). Rather, they were curetted, and the openings cleaned out by pulling gauze through them, to eradicate hair particles. Once the primary pit is dealt with, the secondary pits should heal, because the hairs in them should make their way out. Cleft lift technique with primary closure added the following points; (1) The 'ellipse 'should be marked out on the operating table by first marking two points on one side (the most diseased side), a distance of $1.5-2 \mathrm{~cm}$ from the midline and placed far enough apart to allow a gentle curve to be marked out to just cross the midline a few mms, to include the primary pit in the excision. For small sinuses, use $1.5 \mathrm{~cm}$ from the midline. (2) The distance of maximum width of the 'ellipse' is twice the distance the upper and lower points are from the midline $(3-4 \mathrm{~cm})$. This is measured between the marked lines not between the midline and the outer edge. (3) After probing the tracks to work out their extent, the use of methylene blue injection into the midline pit will enable all branches to be easily identified in case any one is severed or opened during the excision. (4) To avoid a 'dog-ear' at the lower end or the wound tending to 'move' towards the midline, a V excision of skin can be taken off the lower end laterally. This will deviate the lower end a little further from the midline when skin is closed. (5) A fine low-pressure suction drain tube is placed in the fat, brought out lateral to the wound. Allow no suture holes or drain hole to appear in the new midline. (6) Use a subcuticular $3 / 0$ prolene suture loosely knotted as a loop (the wound lengthens when the patient sits, so loose suture needed to prevent a 'cheese-cutter' effect). Support it with a few interrupted $3 / 0$ prolene sutures. (7) The goal of the asymmetric incision is to reduce the depth of the gluteal fold, thereby eliminating the frictional forces between the 2 opposing skin edges. Although the use of an incision that crosses the vertical gluteal fold to excise the pilonidal cavity does eliminate a vertical suture line within the gluteal fold, healing times may remain considerable. Also, The use of asymmetrical or oblique elliptical incisions in an attempt to keep incisions out of the natal cleft where wound healing is poor and to prevent unnecessary tension on the closure of the wound.

The advocates of excision and primary closure of the wound using different techniques emphasize on quicker healing time, fewer postoperative visits and shorter time off work. Those favoring simple excision or lay-open technique, are of the opinion that if general patient satisfaction, period of hospitalization and the recurrence rate are the criteria. Incidences of wound dehiscence following excision and primary repair are much more than previously thought. These procedures also require a long hospital stay and long periods off work.

Our findings suggest that deep tissue damage, although obvious, is not the primary source of none healing, but rather is a secondary effect. Thus, the primary source of surgical failures is not week deep midline tissues but rather the shape of the gluteal cleft, which creates the moist, warm, bacteria-friendly environment. This new paradigm of epidermal origin of pilonidal 
disease has important implications for initial management of the disease. Armstrong \& Barcia, Rickles, and Theodoropoulos et al. all left tissues in place as we did in this study, with similar success.

\section{Conclusion:}

In summary, the goal for treatment of pilonidal disease is 2-fold. The first is excising and healing with a low rate of recurrence. The second is minimizing patient inconvenience and morbidity after the surgical procedure and avoiding hospitalization with loss of workdays. The suggested modified Bascom technique (Cleft lift with primary closure) is an attractive, safe, easily performed operation with minimal morbidity and can be reliably used as a second-line surgical option for recurrent pilonidal disease.

\section{References:}

1- SoendenaaK: Patient characteristics and symptoms in chronic pilonidal sinus disease. Int J Colorectal Dis 1995; 10: 39-42.

2- HullTl: Pilonidal disease. Surg Clin North Am 2002; 82: 1169-1185.

3- Hølmebakk T, Nesbakken A: Surgery for pilonidal disease. Scand J Surg 2005; 94: 43-46.

4- Bascom JU: 'Failed pilonidal surgery'. Arch Surg 2002; 137: 1146-1150.

5- Petersen S, Koch R, Stelzner S, Wendlandt T-P, ludwig K: Primary closure techniques in chronic pilonidal sinus. Dis Colon Rectum 2002; 45: 1458-1467.

6- Karydakis GE: Easy and successful treatment of pilonidal sinus after explanation of its causative process. Aust N Z J Surg 1992; 62: 385-389.

7- Bascom JU: Repeat pilonidal operations. Am J Surg 1987; 154:118-121.

8- Bascom JU: Failed pilonidal surgery. New paradigm and new operation leading to cures. Arch Surg 2002; 137: 1146-1150

9- Bascom J: Utility of the cleft lift procedure in refractory pilonidal disease. Am J Surg 2007; 193: 606-609.

10-Bascom JU: Pilonidal disease: Origin from follicles of hairs and results of follicle removal as treatment. Surgery 1980; 87: 567-572.

11-Chaudhuri A: Single-dose metronidazole vs 5-daymulti-drug antibiotic regimen in excision of pilonidal sinuses with primary closure: A prospective, randomized, double-blinded pilot study. Int J Colorectal Dis 2006; 21: 688-692.

12-Chaudhuri A: Single-dose metronidazole vs 5-daymulti-drug antibiotic regimen in excision of pilonidal sinuses with primary closure: A prospective, randomized, double-blinded pilot study. Int J Colorectal Dis 2006; 21: 688-692.

13-Sakr M: The effect of obesity on the results of Karydakis technique for the management of chronic pilonidal sinus. Int J Colorectal Dis 2003; 18: 36-39.

14-Irfan S: High body mass index as a possible risk factor for pilonidal sinus disease in adolescents. World J Surg 2005; 29: 469-471.

15-Gencosmanoglu R: Modified lay-open (incision, curettage, partial lateral wall excision and marsupialization) versus total excision with primary closure in the treatment of chronic sacorcoccygeal pilonidal sinus. Int J Colorectal Dis 2005; 20: 415-422.

16-Keshava A: Karydakis flap repair for sacrococcygeal pilonidal sinus disease: How important is technique? ANZ J Surg 2007; 77: 181-183.

17-Aldean I: Simple excision and primary closure of pilonidal sinus: A simple modification of conventional technique with excellent results. Colorectal Dis 2005; 7: 81-85.

18-Menteso: Management of pilonidal sinus disease with oblique excision and primary closure: Results of 493 patients. Dis Colon Rectum 2005; 49: 104-108.

19-Saray A, Dirlik M, Caglikulekci M , Turkmenoglu O: Gluteal V-Y advancement fasciocutaneous flap for treatment of chronic pilonidal sinus disease. Scand $J$ Plast Reconstr Surg Hand Surg 2002; 36 (2): 80-84.

20-Arumugam PJ, Chandrasekaran TV, Morgan AR, Beynon J, Carr ND: The 
rhomboid flap for pilonidal disease. Colorectal Dis 2003; 5 (3): 218-221.

21-Bascom J, Bascom T: Failed pilonidal surgery: New paradigm and new operation leading to cures. Arch Surg 2002; 137 (10): 1146-1150.

22-Iesalnieks I, Furst A, Rentsch M, Jauch KW: Primary midline closure after excision of a pilonidal sinus is associated with a high recurrence rate. Chirurg 2003; 74 (5): 461-468.

23-Theodoropoulos GE, Armstrong J, Barcia W, Rickles: Modified Bascom's asymmetric midgluteal cleft closure technique for recurrent pilonidal disease: Early experience in a military hospital: Dis Colon Rectum 2003; 46 (9): 1286-1291. 